\title{
Assessment of the risk factors for impending fractures following radiotherapy for long bone metastases using CT scan-based virtual simulation: a retrospective study
}

\author{
Zuzana Tatar ${ }^{1,2,5^{*}}$, Martin Soubrier ${ }^{2}$, Anne Françoise Dillies ${ }^{1}$, Pierre Verrelle ${ }^{3}$, Stéphane Boisgard ${ }^{4}$ \\ and Michel Lapeyre ${ }^{3}$
}

\begin{abstract}
Background: Radiotherapy for long bone metastases (RTLB) can be complicated by fractures, which considerably increase morbidity and mortality. The aim of this study was to analyze the risk factors for impending fractures following radiotherapy for long bone metastases (RTLB) using CT scan-based virtual simulation.

Methods: Forty-seven (47) patients were treated with RTLB (18 lung, 11 breast, 10 prostate and 8 other cancers) for a period of 18 months. Two doctors analyzed the CT images prior to radiation therapy. The impending fractures were then monitored and the correlation between bone scan parameters and fracture occurrence was analyzed.

Results: The male gender ratio was 0.57 and the mean age 62.8 (33-93) years. The average size of the metastatic lesions was $32(8-87) \times 2(6-81) \times 52(7-408) \mathrm{mm}$ with cortical involvement $(\mathrm{Cl})$ in $66 \%$ of cases. The site was in the upper third of the bone in $92 \%$ of cases (28 femoral, 17 humeral and two tibial).

Ten fractures occurred: two during RTLB, seven after one month and one after 6.6 months. The fractured lesions measured $48(17-87) \times 34(12-66) \times 76(38-408) \mathrm{mm}$. The predictive parameters for fracture were osteolytic (39\% vs. $10 \% ; p=0.02)$ and permeative lesions ( $42 \%$ vs. $0 \% ; p<0.0005)$, a Mirels score $\geq 9(42 \%$ vs. $0 \% ; p<0.0005)$, circumferential $\mathrm{Cl} \geq 30 \%$ (71\% vs. $0 \%, \mathrm{p}<0.00001), \mathrm{Cl} \geq 45 \mathrm{~mm}$ in height $(67 \%$ vs. $0 \%, \mathrm{p}<0.00001)$ and $\mathrm{Cl}$ in thickness $=100 \%$ ( $40 \%$ vs. $0 \% ; p=0.0008)$. In the multivariate analysis, circumferential $\mathrm{Cl} \geq 30 \%$ was the only predictive parameter for fracture $(p=0.00035 ; O R=62 ; C l 95 \%$ : 6.5-595). Overall survival was $91 \%$ and $40 \%$ at one month and twelve months respectively.
\end{abstract}

Conclusions: Prophylactic primary fixation surgery should always be considered when the circumferential $\mathrm{Cl} \geq 30 \%$. Keywords: Long bone metastases, Fractures, Radiotherapy

\section{Introduction}

The aim of extracorporeal radiotherapy of the long bones (RTLB) is to provide control of symptoms, destroy cancer cells in the treated area and prevent malignant disease-related fractures. The analgesic potential of RTLB has been demonstrated in numerous trials, with

\footnotetext{
* Correspondence: ztatar@chu-clermontferrand.fr

${ }^{1}$ Medical Oncology Department, Centre Jean Perrin, Centre de Lutte contre le Cancer de la Région Auvergne, 58 Rue Montalembert, BP 392,

Clermont-Ferrand 63011, France

${ }^{2}$ Rheumatology Department, CHU Gabriel Montpied, 58 Rue Montalembert, BP 69, Clermont-Ferrand 63003, France

Full list of author information is available at the end of the article
}

overall response rates of $60 \%$, including $23-24 \%$ complete responses $[1,2]$. Bone radiotherapy is also a useful means of halting tumor proliferation and then triggering osteoblastic activity with osteoproliferation [3]. Bone recalcification after RTLB has been observed in $70 \%$ of cases, particularly in the fractionated group [3]; recalcification commenced from the first month after RTLB and peaked at three months [4]. Nonetheless, RTLB does not entirely eliminate the risk of fracture [3], particularly in the three months immediately after radiotherapy. A pathologic fracture may occur during this 
time. While such fractures may be atraumatic, they can also considerably aggravate morbidity and mortality.

Prophylactic surgery (followed by RTLB) should be discussed for patients with a high risk of fracture. RTLB after surgery improves bone recalcification and guarantees the stability of the new bone $[5,6]$. While the primary aim of fracture risk assessment is prevention, such an assessment also reduces the risk of surgical overtreatment in patients whose life expectancy is sometimes limited.

Numerous studies [1,7] have explored the risk of fracture using radiographic images (standard $\mathrm{x}$-rays) to determine predictive factors. Analysis of the dimensions of metastatic lesions, and especially any cortical involvement, on standard $\mathrm{x}$-rays alone remains insufficiently predictive of the fracture risk. A three-dimensional CT study provides a more precise assessment of the risk of pathologic fracture, but is not always carried out when the pain is so great that radiotherapy is urgently required. CT scanbased virtual simulation is therefore a valuable tool for providing a precise analysis of tumor infiltration and osteolysis.

The aim of our study was to use CT scan-based virtual simulation to assess the risk of fracture and identify predictive factors with a view to offering prophylactic fixation to those most at risk.

\section{Materials and methods Study design}

This was a retrospective study conducted in a single center. All patients requiring analgesic radiotherapy for long bone metastasis were included. For 18 months, 47 patients were treated with RTLB (18 lung, 11 breast, 10 prostate, 8 other cancers). They had undergone a CT scan-based virtual simulation prior to radiotherapy for a long bone metastatic lesion and were enrolled between September 2010 and February 2012. Two doctors analyzed the scans before irradiation. The impending fractures were then monitored and the correlation between bone scan parameters and fracture occurrence was analyzed. All patients were seen for follow-up and treated solely at our center. Follow-up ended in June 2012: each patient had been monitored for a minimum of four months in order to screen for post-RTLB fractures. Recalcification commenced from the first month after RTLB and peaked at three months [4]; therefore, the fracture risk was considered low after the third month.

\section{Data collected}

Using medical records, we recorded each patient's medical history, primary cancer histological type with staging, RTLB procedures, fractures and disease course. The target lesion was documented with standard $\mathrm{x}$-rays, bone scintigraphy or a diagnostic CT scan.
The virtual simulation was carried out using a 16-slice GE scanner with an $80 \mathrm{~cm}$ ring no more than three weeks prior to the start of radiotherapy. The total dose, fractionation, X-ray energy and interval between sessions varied depending on the general condition of the patient, the intensity of pain experienced lying on the scanner bed and technical constraints.

Two doctors (a radiotherapist and an oncologist) systematically analyzed several parameters that are known to be risk factors for pathologic fractures from previous publications [8-24]: the type and appearance of a metastasis, the mean dimensions of the lesion and the cortical involvement (CI) (craniocaudal, circumferential and thickness [Figure 1]):

- Type of metastasis: a lesion was considered to be "well defined" if its external margins were identifiable in all three spatial planes and "diffuse" if its margins were difficult to identify in the threedimensional analysis because of an infiltrative appearance.

- - Appearance of metastasis: lesions were divided into five categories. They could be "normal" (normal appearance of the bone on the scan), "osteolytic" (a primarily lytic lesion with a decrease in bone density), "osteoblastic" (a mainly blastic lesion with an increase in bone density), "mixed" (both lytic and blastic features) or "moth-eaten" (homogeneous "chewed", infiltrated appearance).

- The mean dimensions of the metastatic lesion were also measured (height, diameter in the transverse plane and $\mathrm{CI}$ ). Lesion height $(\mathrm{mm})$ was assessed by measuring the difference between the outermost transverse slice and the innermost slice on which the lesion could be seen, and then multiplying this difference by the thickness of the CT slices. "Diameter 1" ( $\mathrm{mm}$ ) was the length of the widest axis of the metastatic lesion in the transverse plane. "Diameter 2" ( $\mathrm{mm}$ ) was the largest dimension measured perpendicular to "Diameter 1" in the transverse plane. Circumference was measured in the area considered to be most at risk of fracture by both observers. Circumference perimeter was the measurement of the external perimeter of the cortical bone $(\mathrm{mm})$ in the most at-risk area.

- Cortical thickness (mm) was the measurement of the thickness of the cortex considered to be normal in the most at-risk area.

- Craniocaudal cortical lysis $(\mathrm{mm})$ was the measurement of the maximum $\mathrm{CI}$ height in the craniocaudal plane. The $30 \mathrm{~mm}$ threshold involvement was always recorded since this is the threshold predictive of pathological fracture according to several authors $[11,22,23]$. 


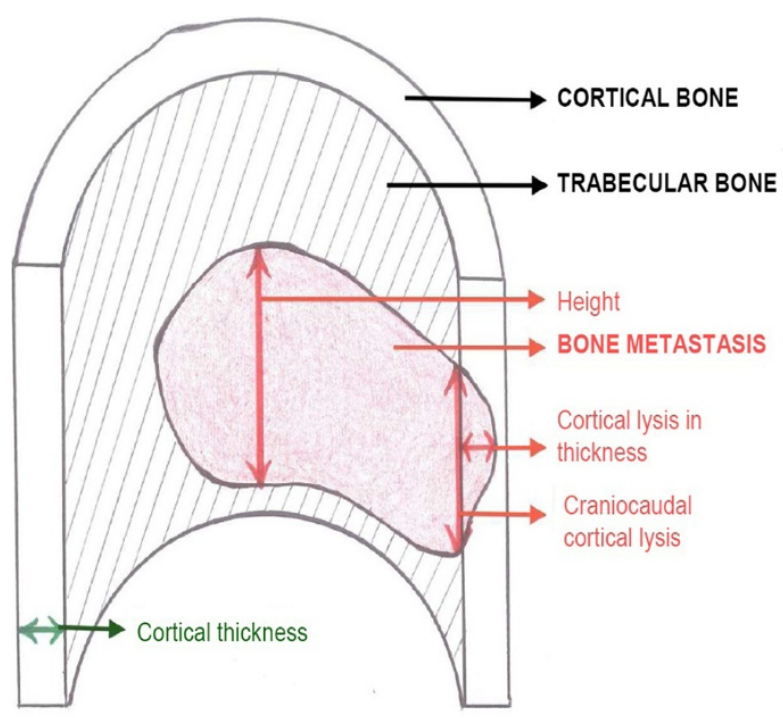

Transversal slice of a bone diaphysis

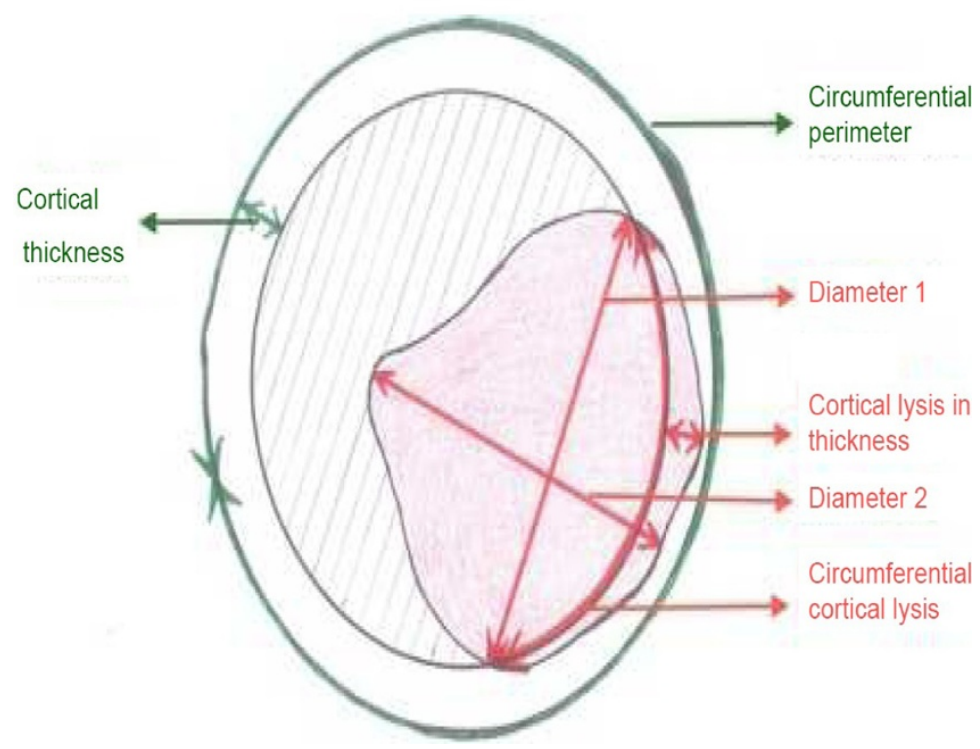

Figure 1 Diagrammatic slice of a bone diaphysis.

- Circumferential cortical lysis (mm) was the measurement of the diseased cortex perimeter in the most at-risk area. The circumferential lysis percentage was systematically determined by calculating the ratio of circumferential cortical lysis to the circumferential perimeter of the bone. The threshold involvement of $50 \%$ was always recorded since this is the threshold predictive of pathologic fracture according to several authors [9,10,15-17,21-23].
- Cortical thickness lysis (mm) was the measurement of the maximum thickness of cortical lysis in the at-risk area. The percentage of cortical lysis thickness was always determined.

- The Mirels score takes into account anatomical location, extent of cortical lysis, appearance of the lesion and pain intensity [21] and was calculated for each metastatic lesion. A Mirels score of nine or more was found to be predictive of fracture. 
- If two lesions were present at the same time, only the lesion with the highest risk of fracture was measured.

\section{Statistical analysis}

The statistical analysis was conducted using the $C h i^{2}$ test and means comparison test. The performance characteristics of the craniocaudal and circumferential cortical lysis thresholds were analyzed using the ROC curve.

Logistic regression generalized linear modeling was used for the multivariate analysis.

The overall survival and fracture incidence curves were calculated using the Kaplan-Meier method. A Log-Rank test was used to compare the survival curves.

The value of $\mathrm{p}$ was considered significant when $<0.05$.

\section{Results}

Between September 2010 and February 2012, 37 patients with 47 lesions ( 28 femoral, 17 humeral and two tibial) underwent analgesic radiotherapy for long bone metastasis. The patients had been monitored for a minimum of four months. The male gender ratio was 0.59 and the mean age was 62.8 years (33-93). Cancer staging was I-II for $56.8 \%$ and II-III for $43.2 \%$. The primary cancers were lung (35.1\%), prostate (27\%), breast (16.3\%) and others (21.6\%). There were 32 adenocarcinomas, two squamouscell cancers and three other types. At the moment their painful long bone metastasis was discovered, 18 patients had been receiving treatment with bisphosphonates and 25 with chemotherapy. Surgery was not initially performed for a variety of reasons, including poor general patient health, increase in pain refractory to medical treatment requiring urgent radiotherapy and low risk of impending fracture. Twenty-two [22] of the 47 lesions received a single dose ( 7 to $8 \mathrm{~Gy}$ ). The 25 other lesions received 15 to 30 Gy in three to ten sessions over three to 19 days. The radiation dose was delivered through hard X-ray energy (5.5 to $18 \mathrm{MV}$ ).
Ten of the 47 radiated lesions fractured during or after RTLB. When overall survival is taken into account, the incidence of fractures was $20 \%$ one month after RTLB and $25.9 \%$ at the end of the study. Two fractures occurred during RTLB, and another seven occurred in the first thirty days. The last fracture occurred at 6.6 months (Figure 2).

\section{Factors predictive of fracture}

In the univariate analysis (Table 1), the primary cancer type, histological type, prior treatments (chemotherapy, hormone therapy, targeted therapies and radiotherapy), symptomatic treatments (bisphosphonates and corticosteroids) were not predictive of fractures. We found no statistically significant difference in anatomical lesion location (upper, middle or lower third; $\mathrm{p}=0.85$ ), bone type (femur, humerus, tibia), existence of local predisposing factors or radiation treatment schedule. There was no correlation between radiotherapy schedule and bone fracture: the radiotherapy procedures were the same in both groups, with or without fractures (Table 2).

The risk factors for impending fracture were as follows: an osteolytic ( $39 \%$ vs. $10 \%$; $\mathrm{p}=0.02)$ and diffuse appearance $(42 \%$ vs. $0 \% ; \mathrm{p}<0.0005)$, circumferential $\mathrm{CI} \geq 50 \%$ $(80 \%$ vs. $5 \%, \mathrm{p}<0.00001)$ and $\geq 30 \%(71 \%$ vs. $0 \%, \mathrm{p}<$ $0.00001)$, height of involvement $\geq 30 \mathrm{~mm}(48 \%$ vs. $0 \%, \mathrm{p}<$ $0.00001)$ and $\geq 45 \mathrm{~mm}(67 \%$ vs. $0 \%, \mathrm{p}<0.00001)$ and cortical thickness $=100 \%$ ( $38 \%$ vs. $0 \% ; \mathrm{p}=0.0008)$. A Mirels score $\geq 9(42 \%$ vs. $0 \% ; \mathrm{p}<0.0005)$ was also predictive of fracture.

The craniocaudal and circumferential involvement thresholds with the greatest sensitivity and specificity in the study population were $\geq 45 \mathrm{~mm}$ for craniocaudal involvement and $\geq 30 \%$ for circumferential involvement (Figure 3).

In the multivariate analysis, only circumferential involvement $\geq 30 \%$ was predictive of fracture ( $\mathrm{p}=0.00035$; $\mathrm{OR}=62$; CI $95 \%=[6.45-595])$.

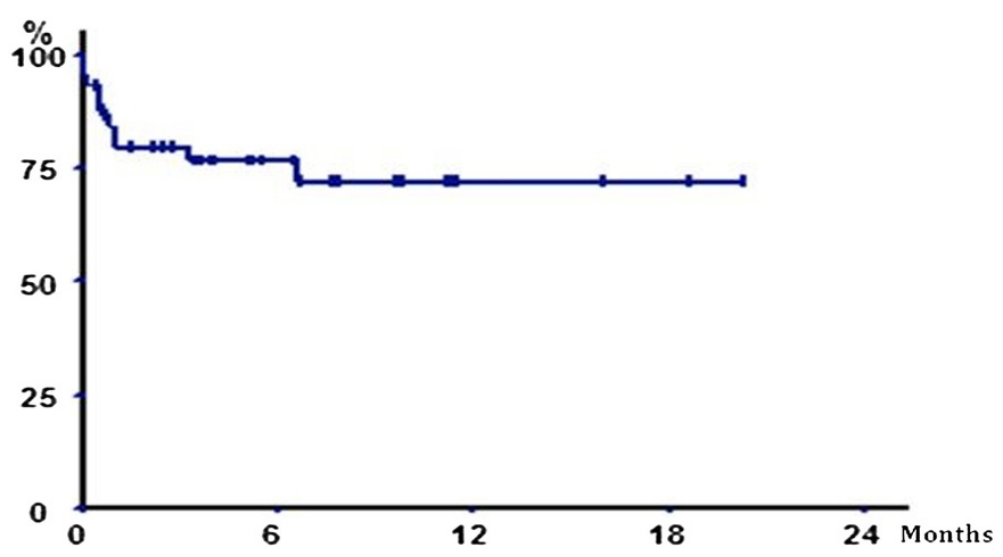

Figure 2 Probability of fracture-free survival following RTLB (Kaplan-Meier). 
Table 1 Comparison of characteristics, group with fracture versus group without fracture (Chi 2 test, comparison of means) a) Clinical parameters

\begin{tabular}{|c|c|c|c|c|}
\hline & Total (\%) & Fractures (\%) & Without fractures (\%) & \\
\hline Number of patients & 37 & 10 & 27 & \\
\hline Number of lesions radiated & 47 & 10 & 37 & \\
\hline Age & & & & $p=0.16$ \\
\hline Mean (years) & 62.8 & 65.3 & 62.2 & \\
\hline Age range (years) & $33-93$ & $33-87$ & $33-93$ & \\
\hline Gender & & & & NS \\
\hline Men & $27(57.5)$ & $4(14.8)$ & $23(85.2)$ & \\
\hline Women & $20(42.5)$ & $6(30)$ & $14(70)$ & \\
\hline Primary cancer & & & & NS \\
\hline Lung & $18(38.3)$ & $4(22)$ & $14(78)$ & \\
\hline Breast & $11(23.4)$ & $3(27)$ & $8(73)$ & \\
\hline Prostate & $10(21.3)$ & --- & $10(100)$ & $p=0.08$ \\
\hline Other & $8(17.0)$ & $3(37.5)$ & $5(62.5)$ & \\
\hline Corticosteroids & & & & NS \\
\hline$<1$ month & $1(2.1)$ & $1(100)$ & -- & \\
\hline$>1$ month and $<6$ months & $2(4.2)$ & $1(50)$ & $1(50)$ & \\
\hline$>6$ months & 0 & --- & -- & \\
\hline No & $42(89.5)$ & $8(19)$ & $34(81)$ & \\
\hline NA & $2(4.2)$ & --- & $2(100)$ & \\
\hline Biphosphonates & & & & NS \\
\hline Yes & $21(44.7)$ & $4(19)$ & $17(81)$ & \\
\hline No & $21(44.7)$ & $4(19)$ & $17(81)$ & \\
\hline NA & $5(10.6)$ & $2(40)$ & $3(60)$ & \\
\hline Chemotherapy ongoing & & & & $p=0.03$ \\
\hline Yes & $34(72.3)$ & $10(29)$ & $24(71)$ & \\
\hline No & $13(27.7)$ & --- & $13(100)$ & \\
\hline Long bone & & & & NS \\
\hline Femur & $28(59.6)$ & $7(25)$ & $21(75)$ & \\
\hline Humerus & $17(36.2)$ & $3(18)$ & $14(78)$ & \\
\hline Tibia & $2(4.2)$ & --- & $2(100)$ & \\
\hline Locoregional history & & & & NS \\
\hline Fracture & $2(4.2)$ & $1(50)$ & $1(50)$ & \\
\hline Osteoarthritis & $5(10.5)$ & --- & $5(100)$ & \\
\hline No & $40(85.1)$ & $9(23)$ & $31(77)$ & \\
\hline Contralateral prosthesis & $4(8.5)$ & --- & $4(100)$ & NS \\
\hline Pain intensification & & & & NS \\
\hline Yes & $42(89.4)$ & $10(24)$ & $32(76)$ & \\
\hline No & $5(10.6)$ & --- & $5(100)$ & \\
\hline \multicolumn{5}{|l|}{ a) Radiological parameters } \\
\hline Number of lesions radiated & 47 & 10 & 37 & \\
\hline Localization & & & & NS \\
\hline Upper third & $43(91.5)$ & $9(21)$ & $34(79)$ & \\
\hline Lower third & $4(8.5)$ & $1(25)$ & $3(75)$ & \\
\hline
\end{tabular}


Table 1 Comparison of characteristics, group with fracture versus group without fracture (Chi 2 test, comparison of means) a) Clinical parameters (Continued)

Type of metastatic spread

Well defined

Diffuse

Appearance of the metastatic lesion

Normal

Osteolytic

Mixed

Osteoblastic

Moth---eaten

Mean dimensions (mm)

Height
Cl 95\%
Diameter 1
Cl 95\%
Diameter 2
Cl 95\%
Circumferential perimeter
Cl 95\%
Cortical thickness
Cl 95\%

Craniocaudal cortical lysis

Mean $(\mathrm{mm})$

Cl 95\%

Cortical lysis threshold

No

$<30 \mathrm{~mm}$

$\geq 30 \mathrm{~mm}$

Circumferential cortical lysis

Mean (mm)

Cl 95\%

Cortical lysis (\%)

No

$<50 \%$

$\geq 50 \%$

Cortical lysis in thickness

$\begin{array}{ll}\text { Mean }(\mathrm{mm}) & 2.2 \\ \text { Cl } 95 \% & (1.6 ; 2.9) \\ \text { Cortical lysis (\%) } & \\ \text { No } & 16(34.0) \\ 0-99 \% & 5(10.7) \\ 100 \% & 26(55.3)\end{array}$

Mirels score

$$
\leq 7
$$$$
=8
$$$$
\geq 9
$$

\section{$23(49.0)$}

$24(51.0)$

$2(4.2)$

$18(38.3)$

$15(31.9)$

$11(23.5)$

$1(2.1)$

59.8

$(40.5 ; 79.1)$

32.2

$(26.8 ; 37.5)$

22.9

(18.6; 27.3)

142.6

(129.9; 155.3)

3.5

(3.1; 3.9)

45.2

$(25.3 ; 65.0)$

16(34.0)

$10(21.3)$

$21(44.7)$

32.3

(22.3; 42.3)

$16(34.0)$

$21(44.7)$

$10(21.3)$

2.2

6 (34.0)

26 (55.3)

$14(29.8)$

9 (19.1)

$24(51.1)$

$---$

$10(42)$

$--$

7 (39)

3 (20)

---

$--$

80.07

(50.37; 109.8)

48.5

$(41.8 ; 55.1)$

34.2

(29.5; 38.8)

133.1

$(127.1 ; 139.1)$

3.3

(3.2; 3.4)

103.0

(85.6; 120.4)

$---$

$--$

10 (47.6)

78.6

$(74.5 ; 82.6)$

$--$

$2(9.5)$

8 (80)

3.3

(3.2; 3.4)

$--$

$--$

$10(38.5)$

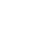

$---$

---

$10(42)$

$$
\begin{aligned}
& 23(100) \\
& 14(58) \\
& 2(100) \\
& 11(61) \\
& 12(80) \\
& 11(100) \\
& 1(100)
\end{aligned}
$$
$p=0.0005$

$$
p=0.02
$$

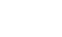

46.1

(33.3; 58.9)

$$
p<0.01
$$

27.6

(22.7; 32.6)

$p=0.01$

19.8

(15.3; 24.3)

$p=0.01$

145.2

$p=0.15$

(130.3; 160.1)

3.6

$p=0.27$

$(3.1 ; 4.0)$

$p<0.0001$

28.6

(14.7; 42.6)

$16(100)$

$10(100)$

11 (52.4)

$p<0.0001$

19.8

(11.6; 28.1)

$16(100)$

19 (90.5)

2 (20)

$p=0.0018$

2.0

(1.2; 2.7)

16 (100)

5 (100)

$16(61.5)$

$P=0.0005$

Cl: confidence Interval; NA: not applicable; NS: not significant. 
Table 2 Comparison of fractionation type, group with fracture versus group without fracture

\begin{tabular}{llll}
\hline & N (\%) & Fractures (\%) & $\begin{array}{l}\text { Without } \\
\text { fractures (\%) }\end{array}$ \\
\hline $\begin{array}{l}\text { Number of lesions radiated } \\
\text { Dose received }\end{array}$ & 47 & 10 & 37 \\
Single fraction & $22(46.8)$ & $5(22.7)$ & $17(77.3)$ \\
7 Gy & $3(6.4)$ & --- & $3(100)$ \\
8 Gy & $19(40.4)$ & $5(26.3)$ & $14(73.7)$ \\
Multiple fractions & $25(53.2)$ & $5(20)$ & $20(80)$ \\
15 Gy & $1(2.1)$ & -- & $1(100)$ \\
20 Gy & $13(27.7)$ & $3(23.1)$ & $10(76.9)$ \\
25 Gy & $3(6.4)$ & --- & $3(100)$ \\
30 Gy & $8(17.0)$ & $2(25)$ & $6(75)$ \\
\hline
\end{tabular}

\section{Overall survival}

The mean follow-up was 5.95 months (0.43-20.27 months) for the 37-patient study population. No patients were lost to follow-up.

Overall survival was $91 \%, 55 \%$ and $40 \%$ at one month, six months and one year respectively (Figure 4). Overall survival was significantly lower in the patients presenting with fractures $(\mathrm{p}=0.014)$.

\section{Discussion}

Our study showed that more than $25 \%$ of patients undergoing radiotherapy for metastases presented with a fracture. In the multivariate analysis, the only factor predictive of fractures identified during the CT-scan-based virtual simulation study was circumferential a CI $\geq 30 \%$.

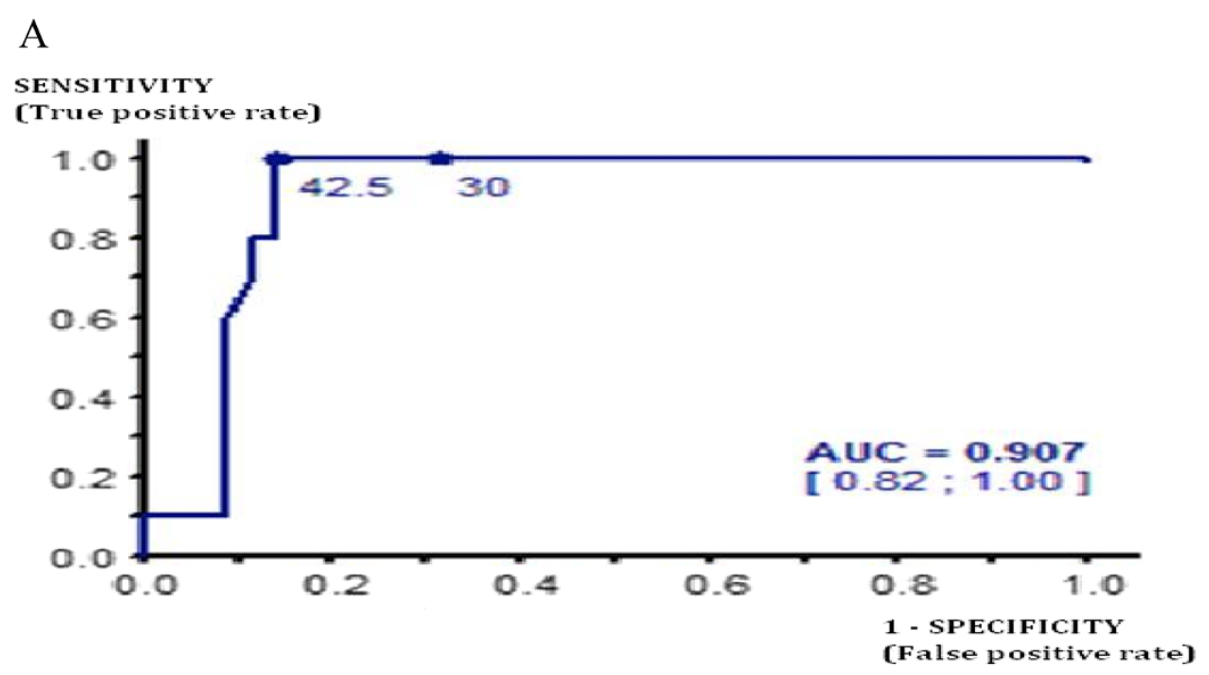

B

SENSITIVITY

CTrue positive rate

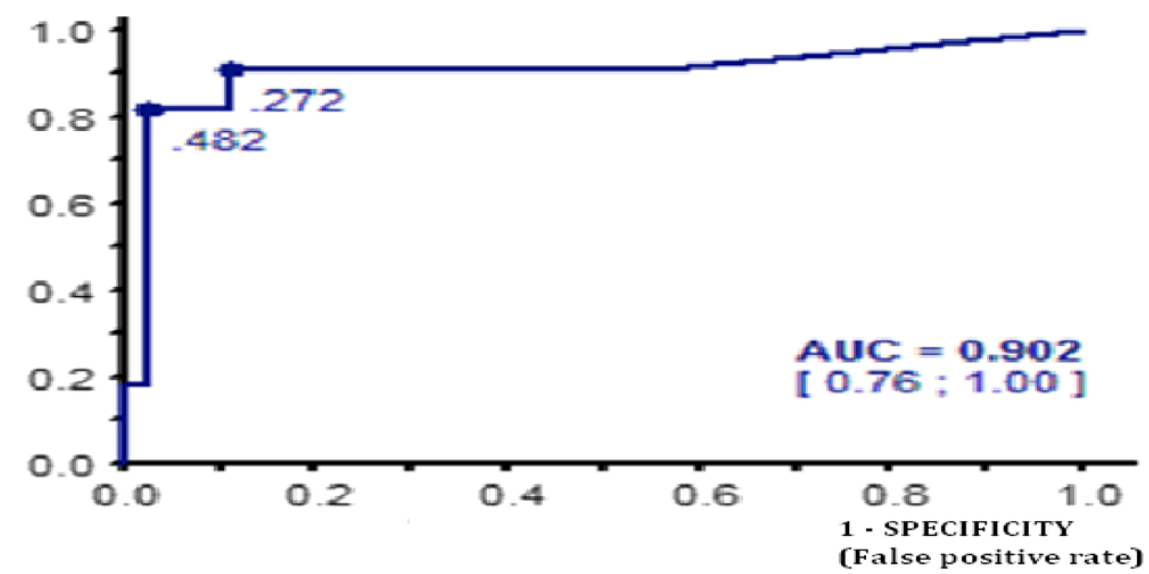

Figure 3 ROC curve. A) Performance of the craniocaudal cortical lysis threshold. No risk of fracture if the craniocaudal cortical involvement is less than $42.5 \mathrm{~mm} .45 \mathrm{~mm}$ threshold: sensitivity $=100 \%$, specificity $=85.7 \%$, positive predictive value $=66.7 \%$, negative predictive value $=100 \%$. B) Performance of the circumferential cortical involvement threshold. No risk of fracture if the circumferential cortical involvement is less than $27 \% .30 \%$ threshold: sensitivity $=100 \%$, specificity $=89 \%$, positive predictive value $=71 \%$, negative predictive value $=100 \%$. 


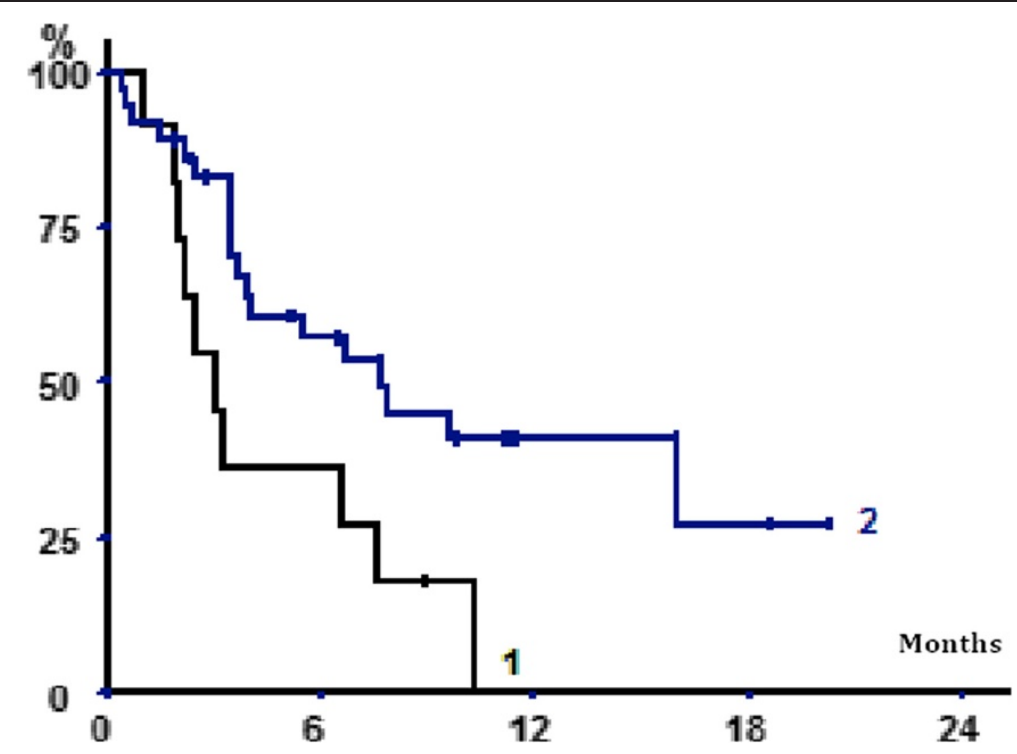

Figure 4 Overall survival after RTLB as a function of fracture incidence. Curve 1: with fracture. Curve 2: without fracture Overall survival curve, Kaplan-Meier; Log--Rank, $p=0.014$.

Several studies [1,7] have explored risk factors for fractures using radiographic images (standard X-rays) to determine predictive factors. In general, high impending fracture risk lesions are lytic [8-17], proximal [10,18,19], large $(>25 \mathrm{~mm})[8-10]$ and femoral (Harrington criteria, $[10,25])$. They trigger increasing pain [8-11,13-15,17,20,21], involve more than $50 \%$ of the cortex circumference [9,10,15-17,21-23] with craniocaudal cortex involvement of over $30 \mathrm{~mm}$ [11,22-24] and a Mirels score of $\geq 9$ [21].

As in other studies, we found that the other radiological parameters predictive of fracture were a lytic, diffuse, poorly circumscribed appearance and cortical involvement (30\% circumferential, $45 \mathrm{~mm}$ height and 100\% thickness). In our study, $\geq 30 \mathrm{~mm}$ femoral craniocaudal cortical involvement was a significant predictor of fracture risk in the univariate analysis, but this consideration could have led to 14 unnecessary surgical procedures $(37.8 \%$ false positive rate).

In our series, a composite Mirels score of $\geq 9$ was also predictive of fracture. However, while this score has the advantage of being very sensitive, it lacks specificity $[7,23,24]$. Therefore, in our study this score was $\geq 9$ in $100 \%$ of the fracture cases, but there were 14 false positives (37.8\%). The three-dimensional study clearly provides a more precise assessment of the risk of pathologic fracture. Measuring circumferential cortical involvement enhances this assessment: primary surgical fixation should be considered in patients with circumferential cortical involvement $\geq 30 \%$.

Our study was representative and comparable with those reported in other series in the literature in terms of age, gender, primary cancer type, performance status and fracture rates [6,15,24,26-28]. In our series, radiotherapy procedures and fractionation did not affect the fracture incidence and the data in the literature are discordant on this point $[26,28]$. Furthermore, in our population, bisphosphonate administration did not influence the risk of bone fractures.

Nevertheless, our study has several limitations:

- It is retrospective in design and our population was very small. However, all the patients treated with RTLB were enrolled and none were lost to followup, which represents a real-life experience.

- The four-month follow-up period could also be seen as a limitation of our study. However, $90 \%$ of the fractures occurred in the month following RTLB and it has been shown that bone recalcification is obtained three months after RTLB, after which time the risk of fractures is very low [4].

- While our scan images were read by a radiotherapist and an oncologist rather than a radiologist, these are the healthcare professionals who are required to assess fracture risk in patients with painful bone metastases on a daily basis. Our patients were oriented directly for urgent analgesic radiation and a CT scan was performed promptly for the virtual simulation (without evaluation by a radiologist). At the same time, we recorded bone scan parameters for this study.

- Due to the retrospective nature of the study, some risk factors for fracture could not be included, such as assist devices, weight-bearing status, bone density status, smoking status and osteoporosis comorbidity. 
Our data must be confirmed in a prospective study including a much larger series of patients, since it is important to precisely establish when prophylactic fixation is required to reduce morbidity and mortality $[5,6,10,15]$. The development of an instrument that identifies patients who have a relatively high risk of developing such a fracture and therefore should be considered candidates for surgical stabilization is helpful. This strategy could optimize the management of fragile metastatic patients. Elective surgery in patients in good general health is simpler and less risky than an emergency procedure, with more rapid relief of pain and recovery of mobility $[29,30]$. Surgical overtreatment also unnecessarily increases morbidity (e.g., hospitalization, general anesthetics and complications arising from a forced supine position) in patients whose life expectancy is limited. The appropriate management of palliative patients and cost-effective proactive approaches may offer more clinical benefit and value for carefully selected patients.

\section{Conclusions}

This study analyzes the risk of fractures following radiation for bone metastasis and attempts to determine which patient population would benefit from prophylactic surgery. Circumferential cortical involvement is easy to measure and should be systematic during CT scanbased virtual simulation prior to radiotherapy.

\section{Consent}

Written informed consent was obtained from the patient for the publication of this report and any accompanying images.

\section{Competing interests}

The authors declare that they have no competing interests.

\begin{abstract}
Authors' contributions
$\mathrm{ZT}$ participated to study design, analysed CT images and participated in the sequence alignment and drafted the manuscript. MS participated in the sequence alignment and drafted the manuscript. AFD participated in the sequence alignment and the reviewing of the manuscript. PV participated in the sequence alignment and the reviewing of the manuscript. SB participated in the sequence alignment and the reviewing of the manuscript. ML participated to study design, analysed $C T$ images and participated in the sequence alignment and drafted the manuscript. All authors read and approved the final manuscript.
\end{abstract}

\section{Author details}

'Medical Oncology Department, Centre Jean Perrin, Centre de Lutte contre le Cancer de la Région Auvergne, 58 Rue Montalembert, BP 392, Clermont-Ferrand 63011, France. ${ }^{2}$ Rheumatology Department, CHU Gabriel Montpied, 58 Rue Montalembert, BP 69, Clermont-Ferrand 63003, France. ${ }^{3}$ Radiotherapy Department, Centre Jean Perrin, Centre de Lutte contre le Cancer de la Région Auvergne, 58 Rue Montalembert, BP 392, Clermont-Ferrand 63011, France. ${ }^{4}$ Orthopedic Surgery Department, CHU Gabriel Montpied, 58 Rue Montalembert, BP 69, Clermont-Ferrand 63003, France. ${ }^{5}$ Département d'Oncologie Médicale, Hôpital de Jour, Centre Jean Perrin, Centre de Lutte contre le Cancer de la Région Auvergne, 58, rue Montalembert, B.P. 392, Clermont-Ferrand 63011, France.
Received: 3 April 2014 Accepted: 2 October 2014

Published online: 16 October 2014

\section{References}

1. Chow E, Harris K, Fan G, Tsao M, Sze WM: Palliative radiotherapy trials for bone metastases: a systematic review. J Clin Oncol 2007, 25(11):1423-1436.

2. Chow E, Zeng L, Salvo N, Dennis K, Tsao M, Lutz S: Update on the systematic review of palliative radiotherapy trials for bone metastases. Clin Oncol (R CollRadiol) 2012, 24(2):112-124

3. Koswig S, Budach V: Remineralization and pain relief in bone metastases after different radiotherapy fractions (10 times 3 Gy vs. 1 time $8 \mathrm{~Gy}$ ). A prospective study. Strahlenther Oncol 1999, 175(10):500-508.

4. Weber W, Rosler HP, Doll G, et al: The percutaneous irradiation of osteolytic bone metastases-a course assessment. Strahlenther Oncol 1992 , 168:275-280.

5. Townsend PW, Rosenthal HG, Smalley SR, Cozad SC, Hassanein RE: Impact ofpostoperative radiation therapy and other perioperative factors on outcome after orthopedic stabilization of impending or pathologic fractures due to metastatic disease. J Clin Oncol 1994, 12(11):2345-2350.

6. Townsend PW, Smalley SR, Cozad SC, Rosenthal HG, Hassanein RE: Role of postoperative radiation therapy after stabilization of fractures caused by metastatic disease. Int J Radiat Oncol Biol Phys 1995, 31(1):43-49.

7. Agarawal JP, Swangsilpa T, van der Linden Y, Rades D, Jeremic B, Hoskin PJ: The role of external beam radiotherapy in the management of bone metastases. Clin Oncol (R Coll Radiol) 2006, 18(10):747-760.

8. Snell W, Beals RK: Femoral Metastases and Fractures From Breast Cancer. Surg Gynecol Obstet 1964, 119:22-24.

9. Beals RK, Lawton GD, Snell WE: Prophylactic internal fixation of the femur in metastatic breast cancer. Cancer 1971, 28(5):1350-1354.

10. Harrington KD: New trends in the management of lower extremity metastases. Clin Orthop Relat Res 1982, 169:53-61.

11. Dijkstra PD, Oudkerk M, Wiggers T: Prediction of pathological subtrochanteric fractures due to metastatic lesions. Arch Orthop Trauma Surg 1997, 116(4):221-224.

12. Bunting R, Lamont-Havers W, Schweon D, Kliman A: Pathologic fracture risk in rehabilitation of patients with bony metastases. Clin Orthop Relat Res 1985, (192):222-227.

13. Bunting RW, Shea B: Bone metastasis and rehabilitation. Cancer 2001, 92(4 Suppl):1020-1028

14. Zickel RE, Mouradian WH: Intramedullary fixation of pathological fractures and lesions of the subtrochanteric region of the femur. J Bone Joint Surg Am 1976, 58(8):1061-1066.

15. Yazawa Y, Frassica FJ, Chao EY, Pritchard DJ, Sim FH, Shives TC: Metastatic bone disease. A study of the surgical treatment of 166 pathologic humeral and femoral fractures. Clin Orthop Relat Res 1990, (251):213-219.

16. Fidler M: Prophylactic internal fixation of secondary neoplastic deposits in long bones. Br Med J 1973, 1(5849):341-343.

17. Parrish FF, Murray JA: Surgical treatment for secondary neoplastic fractures. A retrospective study of ninety-six patients. J Bone Joint Surg Am 1970, 52(4):665-686

18. Miller F, Whitehill R: Carcinoma of the breast metastatic to the skeleton. Clin Orthop Relat Res 1984, 184:121-127.

19. Cheng DS, Seitz CB, Eyre HJ: Nonoperative management of femoral, humeral, and acetabular metastases in patients with breast carcinoma. Cancer 1980, 45(7):1533-1537

20. Hipp JA, Springfield DS, Hayes WC: Predicting pathologic fracture risk in the management of metastatic bone defects. Clin Orthop Relat Res 1995, 312:120-135.

21. Mirels $\mathrm{H}$ : Metastatic disease in long bones. A proposed scoring system for diagnosing impending pathologic fractures. Clin Orthop Relat Res 1989, (249):256-264

22. Menck $H$, Schulze $S$, Larsen E: Metastasis size in pathologic femoral fractures. Acta Orthop Scand 1988, 59(2):151-154.

23. Van der Linden YM, Dijkstra PD, Kroon HM, Lok JJ, Noordijk EM, Leer JW, Marijnen CA: Comparative analysis of risk factors for pathological fracture with femoral metastases. J Bone Joint Surg (Br) 2004, 86(4):566-573.

24. van der Linden YM, Kroon HM, Dijkstra SP, Lok JJ, Noordijk EM, Leer JW Marijnen CA: Dutch Bone Metastasis Study Group. Simple radiographic parameter predicts fracturing in metastatic femoral bone lesions: results from a randomised trial. Radiother Oncol 2003, 69(1):21-31. 
25. Harrington KD: Orthopedicsurgical management of skeletal complications ofmalignancy. Cancer 1997, 80(8 Suppl):1614-1627.

26. Tong D, Gillick L, Hendrickson FR: The palliation of symptomatic osseous metastases: final results of the Study by the Radiation Therapy Oncology Group. Cancer 1982, 50(5):893-899.

27. Steenland E, Leer JW, van Houwelingen $H$, Post WJ, van den Hout WB, Kievit J, de Haes H, Martijn H, Oei B, Vonk E, van der Steen-Banasik E, Wiggenraad RG, Hoogenhout J, Wárlám-Rodenhuis C, van Tienhoven G, Wanders R, Pomp J, van Reijn M, van Mierlo I, Rutten E: The effect of a single fraction compared to multiple fractions on painful bone metastases: a global analysis of the Dutch Bone Metastasis Study. Radiother Oncol 1999, 52(2):101-109.

28. Blitzer PH: Reanalysis of the RTOG study of the palliation of symptomatic osseous metastasis. Cancer 1985, 55(7):1468-1472.

29. Dijstra S, Wiggers T, van Geel BN, Boxma H: Impending and actual pathological fractures in patients with bone metastases of the long bones. A retrospective study of 233 surgically treated fractures. Eur J Surg 1994, 160(10):535-542.

30. Murray JA, Parrish FF: Surgical management of secondary neoplasic fractures about the hip. Orthop Clin North Am 1974, 5(4):887-901.

doi:10.1186/s13014-014-0227-1

Cite this article as: Tatar et al:: Assessment of the risk factors for impending fractures following radiotherapy for long bone metastases using CT scan-based virtual simulation: a retrospective study. Radiation Oncology 2014 9:227.

\section{Submit your next manuscript to BioMed Central and take full advantage of:}

- Convenient online submission

- Thorough peer review

- No space constraints or color figure charges

- Immediate publication on acceptance

- Inclusion in PubMed, CAS, Scopus and Google Scholar

- Research which is freely available for redistribution 